\title{
A 3D Active Surface Model for the accurate Segmentation of Drosophila Schneider Cell Nuclei and Nucleoli
}

\author{
Margret Keuper ${ }^{1,3}$, Jan Padeken ${ }^{2,3}$, Patrick Heun ${ }^{2,3}$, \\ Hans Burkhardt ${ }^{1,3}$, Olaf Ronneberger ${ }^{1,3}$ \\ ${ }^{1}$ Chair of Pattern Recognition and Image Processing, University of Freiburg, \\ Germany \\ ${ }^{2}$ Max-Planck Institute of Immunbiology, Freiburg, Germany \\ ${ }^{3}$ Centre of Biological Signalling Studies (bioss), University of Freiburg, Germany \\ keuper@informatik.uni-freiburg.de
}

\begin{abstract}
We present an active surface model designed for the segmentation of Drosophila Schneider cell nuclei and nucleoli from wide-field microscopic data. The imaging technique as well as the biological application impose some major challenges to the segmentation. On the one hand, we have to deal with strong blurring of the 3D data, especially in z-direction. On the other hand, concerning the biological application, we have to deal with non-closed object boundaries and touching objects. To cope with these problems, we have designed a fully $3 \mathrm{D}$ active surface model. Our model prefers roundish object shapes and especially imposes roughly spherical surfaces where there is little gradient information. We have adapted an external force field for this model, which is based on gradient vector flow $(G V F)$ and has a much larger capture range than standard $G V F$ force fields.
\end{abstract}

\section{Introduction}

For the analysis of living cells, wide-field fluorescence microscopy still plays an important role, because it is prevalently available and, compared to confocal microscopy, has some advantages concerning temporal resolution and phototoxicity. The major disadvantage is the recorded defocused light - volume datasets recorded in wide-field microscopy suffer from strong blurring.

For the analysis of cellular mechanisms, exact knowledge about the subcellular anatomy is necessary. We are looking for a method to accurately detect and segment Drosophila cell nuclei and a subcellular structure, the nucleolus, from three dimensional recordings of cell cultures from a Schneider cell line. Nucleus and nucleolus have been recorded in two separate channels. Channel 1 shows the cell nuclei stained with the fluorescent stain 4',6-diamidino-2-phenylindole (DAPI), channel 2 shows the green fluorescent protein (GFP) stained fibrillarin inside the nucleolus. The voxel size in xy-direction is $0.064 \mu \mathrm{m}$ in z-direction $0.2 \mu \mathrm{m}$. For the segmentation of this data, we are dealing with a variety of problems inherent in 
the biological setting as well as with problems originating from the microscopy technique. In figure 1, an example slice from each channel is displayed, as well as orthogonal slices of one of the nuclei in channel 1. In channel 1, the brighter spots of dense chromatin and the low intensity regions, where there is no chromatin, lead to non-closed boundaries. This and the fact, that there are typically touching cells in the dataset make the segmentation of the nuclei challenging. Additionally, due to artifacts caused by the point spread function, the nucleolus in channel 2 often seems to range outside the nucleus, which, in a biological sense, cannot be the case.

For the detection and segmentation of the nucleoli, the use of all available information in both recorded channels is therefore necessary. We thus designed a preprocessing step, in which we combine the two channels to a Channel Differential Structure. This procedure is inspired by the color differential structure described in [4]. The description of this preprocessing step constitutes the first section of this paper. After this preprocessing step, the segmentation of nuclei and nucleoli are both addressed with a two-step procedure. First, the nuclei are detected by the generalized Hough-Transform [1] for the detection of spherical objects, as it has been used e.g. in [3] for the 3D detection of Arabidopsis thaliana root nuclei in confocal microscopic data and in [2] for the 2D detection of pollen grains in brightfield data. The detection method will be presented in section 3 . The second step, which constitutes the main part of this paper, consists in specifying the objects boundary using a three dimensional active surface model. In the field of biomedical image analysis, active contour methods are widely used for the segmentation and modeling of anatomical structures. The mathematical foundamentals can be found e.g. in [5], a review of deformable models is given e.g. in [6] and [7]. Here, we design a 3D active surface model especially adapted for the segmentation and representation of smooth, 2-sphere like shapes. The grid representation as well as the external forces have been adapted, providing a robust and accurate segmentation with a large capture range even under strong blurring. The detection of the nucleoli is done as for the nuclei with the generalized Hough transform, the segmentation is achieved by the presented active surface model as well - thus proving its generalization ability. Finally, we present the results of our segmentation. These results will be compared to the results that can be achieved with an active surface implementation using a classical gradient vector flow force field and to a standard state of the art method: the user-guided level set implementation ITK-SNAP [8]. For the detection as well as for the segmentation, we decided to use undeconvolved data. Deconvolution makes the images appear clearer in xy-direction but the deconvolution artifacts in z-direction made a good segmentation nearly impossible.

\section{Channel Differential Structure}

For the detection of the nuclei, only channel 1 is used, since here, the boundaries can be seen best (see figure 2(a)). For the nucleoli, the situation is different. 


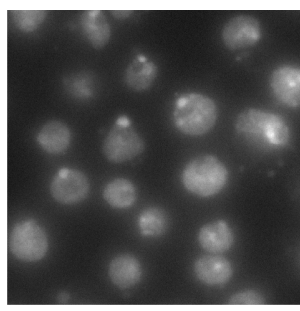

(a)

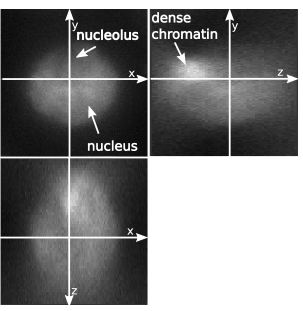

(b)

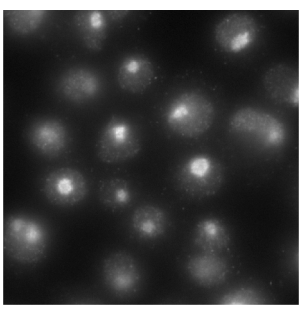

(c)

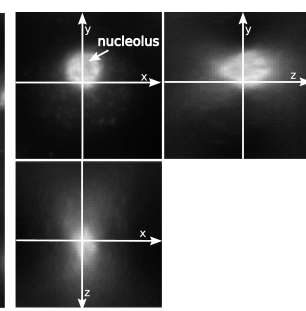

(d)

Fig. 1. Example slices from the two channels of the Drosophila cell raw data and orthogonal slice views of one of the nuclei. (a) and (b): channel 1 shows the cell nuclei stained in DAPI. Brighter regions are caused by denser chromatin. In the location of the nucleoli, no signal is recorded in this channel, thus causing a hole. (c) and (d): in channel 2, the GFP-stained fibrillarin inside the nucleoli has been recorded.

In channel 2 (see figure 2(b)), only the fibrillarin inside the nucleoli has been stained,which results in a bright region inside the nucleolus - but also the whole nucleus can be seen in this channel at a much lower intensity. In channel 1, one can see a hole at the nucleolus' position, which is larger than the stained region in channel 2 - the correct nucleolus boundaries are between the borders of this hole and the fibrillarin in channel 2. Therefore, it makes sense to use the information of both channels for the detection and segmentation of the nucleoli. To do so, the arctangent of the ratio of the intensities in channel $2 I_{c h 2}$ and channel $1 I_{c h 1}$ is computed in each position, yielding some kind of channel diffenrential structure $C D S$. This was inspired by the color differential structure defined in [4]. In [4], this color differential structure is computed as the convolution of the spectral color information with a gaussian derivative to detect gradients between complementary colors.

For our task, not the perceptual color difference is important, which heavily depends on human color perception, but the relation of the channels. The arctangent of the ratio can thus be considered a good distance measure. The twochannel volume data is considered as function $\mathbf{I}: \mathbb{R}^{3} \rightarrow \mathbb{R}_{>0}^{2}$. The $C D S(\mathbf{I})$ can then be computed as

$$
C D S(\mathbf{I})=\nabla\left(\frac{2}{\pi} \arctan \left(\frac{I_{c h 2}}{I_{c h 1}}\right)\right)
$$

where $\nabla=\left(\frac{\partial}{\partial x_{1}}, \frac{\partial}{\partial x_{2}}, \frac{\partial}{\partial x_{3}}\right)$ is the the grad operator and $\arctan \left(\frac{I_{c h 2}}{I_{c h 1}}\right)$ is scalar valued between 0 and $\frac{\pi}{2}$. Positions, where the ratio of the channels has large values are likely to correspond to the nucleolus. The ratio of the intensities inside the nucleus are small, such that the boundaries of nucleus and nucleolus cannot be confounded. Compare figure 2(c). The $C D S(\mathbf{I})$ is used instead of $\nabla I_{c h 2}$ for the further segmentation of the nucleoli. 


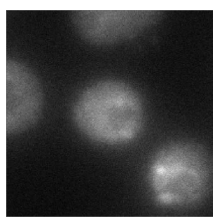

(a) channel 1

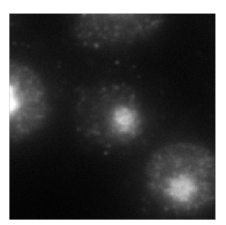

(b) channel 2

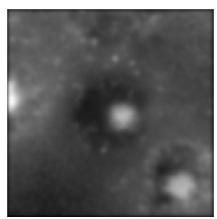

(c) $\frac{2}{\pi} \arctan \left(\frac{I_{c h 2}}{I_{c h 1}}\right)$

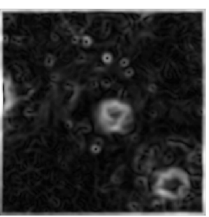

(d) ||$C D S(\mathbf{I}) \|$

Fig. 2. xy-slices from the raw data channels, their ratio and the magnitude of $C D S(\mathbf{I})$.

\section{Detection}

The detection of nucleus and nucleolus is done with the generalized Hough transform for spherical objects [1] as presented in [3]. The main idea is to let each voxel vote for possible positions $\mathbf{c}$ of sphere centers at specific radii $r$.

For this, the dataset has to be smoothed first. We use a Gaussian smoothing with $\sigma_{1}=2 \mu \mathrm{m}$ in all directions. Given the recorded resolution, the resulting estimation for the radii $r$ can therefore not be very precise, but as these radii are used only for the initialization of the active surfaces, these rough estimations are sufficient. Then, one has to select the voting voxels. We choose to use all voxels for the voting and weight the votes with the respective gradient magnitude values. A threshold would not make sense, because the intensity variations of the nuclei within one dataset are too strong. The gradient magnitude and direction of the voxels is used to determine the position of the votes. Finally, the votes are combined by integration. Formally, the four dimensional voting field $P$ of a function $I: \mathbb{R}^{3} \rightarrow \mathbb{R}$ is computed as follows.

$$
P(\mathbf{c}, r)=\int_{\mathbb{R}^{3}} G_{\sigma_{2}}\left(\mathbf{c}-r \frac{\left(\nabla\left(G_{\sigma_{1}} * I\right)\right)(\mathbf{x})}{\left\|\left(\nabla\left(G_{\sigma_{1}} * I\right)\right)(\mathbf{x})\right\|}\right)\left\|\left(\nabla\left(G_{\sigma_{1}} * I\right)\right)(\mathbf{x})\right\| d \mathbf{x}
$$

where $G_{\sigma}$ is the 3D Gaussian distribution with standard deviation $\sigma$ in all directions. $G_{\sigma_{2}}$ is used as an indicator function giving contribution only if the argument is nearby zero.

The detection is then done by determining the local maxima of the voting field $P$.

\section{Active Surfaces}

After the detection step, we have an estimated center $\mathbf{c}$ and radius $r$ for each nucleus and nucleolus, but in reality, both structures are not spherical. Thus, the best radius can only give a very rough estimation of the object's size. An exact segmentation has been done based on this estimation by employing active surface models.

The three dimensional active surface can be described as a function $\mathbf{X}:[0,1] \times$ $[0,1] \rightarrow \mathbb{R}^{3}$ which is placed on a dataset $I: \mathbb{R}^{3} \rightarrow \mathbb{R}$. These active surfaces 
have internal energies, depending only on the shape of the model itself, and are subjected to external energies coming from the underlying dataset to which the model shall be adapted. The total energy of an active surface is thus

$$
E=E_{\text {int }}(\mathbf{X})+E_{\text {ext }}(\mathbf{X}) .
$$

The adaption takes place in minimizing this energy.

\subsection{Implementation}

Mesh Design For the realization of the active surface model in three dimensional space, a good choice of the grid structure is crucial. As we suppose a roughly spherical shape of the objects to detect, it is intuitive to initialize the active surface with a spherical grid. To provide an equidistant sampling of the sphere, an icosahedron can be used as initial structure. We have used subdivisions of the icosahedron, which still provide a nearly equidistant sampling, with a higher resolution, leading to a more accurate segmentation.

As initialization, we used the parameters found in the detection step, i.e. we initialized the grid as a subdivided icosahedron with 162 vertices $v_{i} \in \mathrm{V}$ located at positions $\mathbf{x}_{v_{i}}$ around the center $\mathbf{c}$ of the nucleus or nucleolus. All vertices $v_{i}$ have distance $r$ from the center.
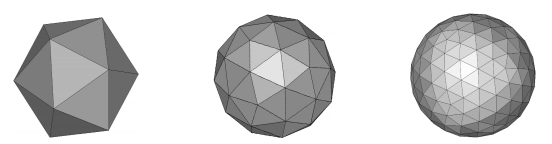

Fig. 3. The sampling of the spherical surface can be done equidistantly by using an icosahedron. Icosahedron subdivisions yield a nearly equidistant sampling.

Mesh Operations To ensure that, during the evolution of the active surface, the resolution of the grid is always high enough, we implement splitting and merging operations. After each iteration, the length $l$ of every edge is checked. Edges longer than a threshold $t_{u}$ are split and a new vertex is inserted. This new vertex has to be connected to all its neighboring vertices. If edges are shorter than threshold $t_{l}$, they are deleted and the corresponding vertices are merged. See figure 4 for an example.

\subsection{Internal Forces}

As internal energy, the weighted first and second derivative of the surface are used, corresponding to the classical continuity and curvature energies:

$$
E_{i n t}=\frac{1}{2} \int_{\mathbf{s}} \alpha\left\|\frac{\partial \mathbf{X}}{\partial \mathbf{s}}\right\|^{2}+\beta\left\|\frac{\partial^{2} \mathbf{X}}{\partial \mathbf{s}^{2}}\right\|^{2} d \mathbf{s}
$$



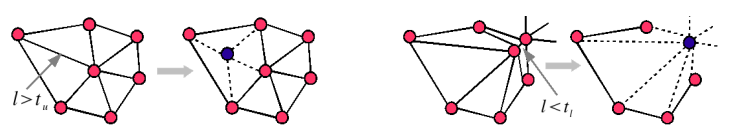

Fig. 4. (left) If the length $l>t_{u}$, the edge is split. (right) Edges for which $l<t_{l}$ are merged.

where $\mathbf{s} \in[0,1] \times[0,1]$ and $\alpha$ and $\beta$ are the weighting coefficients. The minimization of $E$ thus leads to the Euler-Lagrange equation

$$
\frac{\partial}{\partial \mathbf{s}}\left(\alpha \frac{\partial \mathbf{X}}{\partial \mathbf{s}}\right)-\frac{\partial^{2}}{\partial \mathbf{s}^{2}}\left(\beta \frac{\partial^{2} \mathbf{X}}{\partial \mathbf{s}^{2}}\right)-\nabla E_{e x t}=0
$$

where the internal forces $\mathbf{F}_{\text {ela }}:=\frac{\partial}{\partial \mathbf{s}}\left(\alpha \frac{\partial \mathbf{X}}{\partial \mathbf{s}}\right)$ and $\mathbf{F}_{\text {rig }}:=\frac{\partial^{2}}{\partial \mathbf{s}^{2}}\left(\beta \frac{\partial^{2} \mathbf{X}}{\partial \mathbf{s}^{2}}\right)$ prevent the surface from stretching and bending too much. These energies are minimal for planar surfaces. Therefore, on spherical structures, they act as shrinking forces. The internal forces of the active surface have to be adapted for the three dimensional grid implementation. Since we want to preserve the equidistant sampling, the elasticity force can be approximated with

$$
\mathbf{F}_{\text {ela }}\left(v_{i}\right)=\frac{1}{c^{2}}\left(\sum_{j, v_{j} \in N\left(v_{i}\right)} \frac{\mathbf{x}_{v_{j}}-\mathbf{x}_{v_{i}}}{|N(v i)|}\right),
$$

where $N\left(v_{i}\right)$ is the set of all neighbors of vertex $v_{i}$ and $c$ is the average distance between two neighboring vertices

$$
c=|\mathrm{V}| \sum_{i} \sum_{j, v_{j} \in N\left(v_{i}\right)} \frac{\left|N\left(v_{i}\right)\right|}{\left\|\mathbf{x}_{v_{j}}-\mathbf{x}_{v_{i}}\right\|}
$$

$|\mathrm{V}|$ is the cardinality of the set of vertices. The rigidity force corresponding to the forth derivative can by analogy be approximated as

$$
\mathbf{F}_{\text {rig }}\left(v_{i}\right)=\frac{1}{c^{4}} \frac{1}{\left|N\left(v_{i}\right)\right|\left(\left|N\left(v_{j}\right)\right|-1\right)} \sum_{j, v_{j} \in N\left(v_{i}\right)} \sum_{\substack{k, v_{k} \in N\left(v_{j}\right) \\ k \neq i}}\left(4 \mathbf{x}_{v_{j}}-\mathbf{x}_{v_{k}}-3 \mathbf{x}_{v_{i}}\right) \text {. (8) }
$$

\subsection{External Forces}

Since the external forces are responsible for the attraction of the active surface to the underlying data, these forces have to be adapted very carefully to the specific task. A classical choice would be $\mathbf{F}_{\text {ext }}=-\nabla E_{\text {ext }}$ with $E_{\text {ext }}=-\|\nabla I\|^{2}$, but for the segmentation of the nuclei, some application specific challenges are given. As the chromatin displayed in channel 1 is not homogeneous, some blob structures and holes can be seen in the nucleus. Thus, neither the intensity values nor the pure gradient information can be used as an external energy for the segmentation 
of the nuclei. Especially nucleoli lying near the nucleus boundary cause the image gradients to pull the contour inwards into the nucleus. To address this problem, we use external forces based on the gradient information of the data coupled with prior knowledge from the detection step. We assume the nuclei to have a star-shaped surface, i.e. every surface point can be reached from the detected center c. As proposed in [2] for the 2D case in pollen segmentation, we then used a projection of the dataset gradients onto radial vectors pointing away from the detected center $\left(\nabla I_{\text {radial }}\right)(\mathbf{x})=\left\langle(\nabla I)(\mathbf{x}), \frac{\mathbf{x}-\mathbf{c}}{\|\mathbf{x}-\mathbf{c}\|}\right\rangle$, thus reducing the influence of vectors pointing in other directions. Additionally, as done in [2], those vectors originating from darker inner structures and thus pointing outwards were set to zero length. The resulting gradient image contains by far less gradients corresponding to structures other than the nucleus, but the vectors set to zero length still cause problems in the next step.

Instead of applying the Canny edge detector as it was done in [2], we directly use the resulting gradient magnitude as edge information. We compute the gradients of this edge image and, to get rid of the gradients now caused by the zeromagnitude regions, we use the radial projection of these gradients. This results in a vector valued function $\mathbf{A}: \mathbb{R}^{3} \rightarrow \mathbb{R}^{3}$ with

$$
\mathbf{A}(\mathbf{x})=\left\langle\left(\nabla\left(s\left(\nabla I_{\text {radial }}\right)\right)\right)(\mathbf{x}), \frac{\mathbf{x}-\mathbf{c}}{\|\mathbf{x}-\mathbf{c}\|}\right\rangle \cdot \frac{\mathbf{x}-\mathbf{c}}{\|\mathbf{x}-\mathbf{c}\|},
$$

where $\langle.,$.$\rangle is the scalar product and s(x)$ is defined as

$$
s(x)= \begin{cases}x, & \text { if } x>0 \\ 0, & \text { otherwise }\end{cases}
$$

The external force was finally found as the weighted sum of the gradient vector flow $G V F(\mathbf{A})$ (e.g. in [5]) and the radially projected gradients, pulling the surface outside the object. $\nabla I_{\text {radial }}$ counteracts the shrinking effect of the internal forces.

$$
\mathbf{F}_{\text {ext }}=\gamma G V F(\mathbf{A})-\delta \nabla I_{\text {radial }}
$$

$\gamma$ and $\delta$ are the weighting coefficients. The in this way defined external force field has some major advantages compared to standard gradient based force fields. On the one hand, the projection onto the radial vectors promotes 2 -spherical shapes, on the other hand, these projections and the deletion of gradient vectors pointing in the wrong direction has the effect that the capture range of the resulting force field is much larger. This is important, because of the touching cells in the dataset.

\section{$5 \quad$ Experiments and Results}

The method was tested on 45 datasets containing 440 cells. For the detection of the nuclei, we searched for spheres with diameters between 3.6 and $6.4 \mu \mathrm{m}$. Although more or less strong deformations of the nuclei can be observed, a quite 


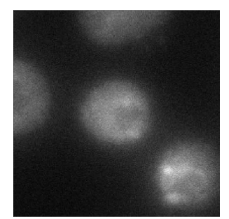

(a)

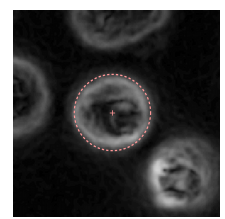

(b)

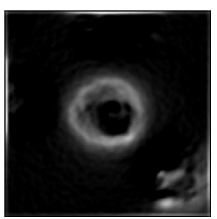

(c)

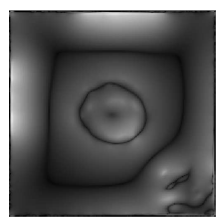

(d)

Fig. 5. (a) xy-slice from the original dataset (b) xy-slice from the gradient magnitude of the data with the estimated center and radius (c) xy-slice from the magnitude of the projected gradients $\left\|\nabla I_{\text {radial }}\right\|$, , (d) xy-slice from the magnitudes of the gradient vector flow $\|G V F(\mathbf{A})\|$.

reliable detection of the nuclei was possible. Nuclei clearly lying on the border of the captured dataset were not detected and left out of the evaluation. Out of 440 nuclei, we have correctly detected 437 nuclei, 3 nuclei have not been detected. There were 16 false positives: 8 nuclei were detected where there was no data and 8 defect cells have been detected as nuclei. For some of the nuclei, the estimated radii were too small (compare figure 6), which results from the relatively strong smoothing. For the detection of the nucleoli, we searched for spheres with diameters between 0.6 and $1.6 \mu \mathrm{m}$. The result of the nuclei segmentation was used as a mask for the detection of the nucleoli, i.e. every nucleolus has to lie inside a nucleus. To ensure this, only the Hough-votes within the nucleus were evaluated and exactly one nucleolus was searched inside each nucleus, since for healthy cells, there should only be one nucleolus. Despite this fact, there are cells in the datasets containing more than one clearly defined nucleolus. Detection results for an example dataset can be seen in figure 6 . Correct nucleoli positions were found in all of the analyzed datasets - only where there was more than one nucleolus inside the same nucleus, one of the nucleoli was missed.

For the accurate segmentation, we manually tested some parameter sets for
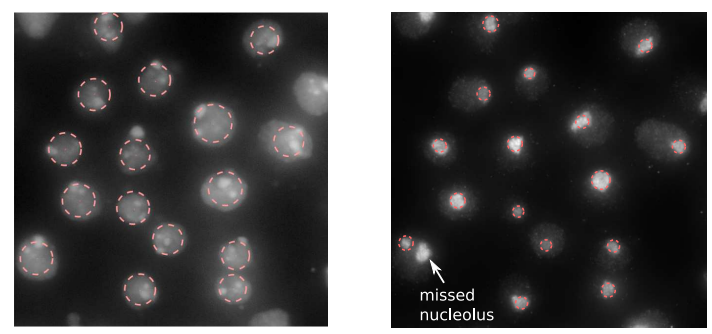

Fig. 6. (left) Detection results of nuclei displayed in the maximum intensity projection (MIP) of channel 1. (right) Detection results of nucleoli in the MIP of channel 2.

three example nuclei and then used the best parameters for the segmentation of all the nuclei. Finally, we used $\alpha=\beta=0.2, \gamma=0.9$, and $\delta=0.7$, but the 
method turned out to be very robust against smaller parameter variations. For the nucleoli, we picked $\alpha=\beta=0.1, \gamma=0.9$, and $\delta=0.5$. With those parameters, satisfying results could be achieved for the segmentation of all nuclei and nucleoli of the dataset. Some randomly chosen example results can be seen in figure 7 .

To evaluate the results of the segmentation, we compared the segmentation carried out with the presented method with the results that could be achieved with an active surface implementation using a standard $G V F$ force field as well as with results from the ITK segmentation tool ITK-SNAP [8], which is based on a level set implementation. The standard $G V F$ force field was computed directly from the image gradients as $G V F(\nabla\|\nabla I\|)$. The ITK-SNAP segmentation implements two algorithms: 3D geodesic active contours, where the internal forces are based on the gradient magnitude in the dataset, and a region competition method, based on voxel probability maps, which are estimated by manually adjusted intensity thresholds. In all cases, it was not possible to find parameters that worked for the whole dataset. For our four example cells, we manually adapted the parameters for each nucleus as good as possible, but even though we could not get good results for all of the cells. The 3D geodesic active contours even did not work at all, because of the blurring in z-direction. It was not possible to find parameters, that prevented the contour from running out of the object in upper and lower dataset regions before filling the nucleus' volume in the center, where there are in fact stronger gradients. For a comparison of the other two methods to our presented method, see figure 7. Although the region competition method from ITK-SNAP in most cases yields acceptable results if the manual threshold is carefully adjusted, our method worked best for all of the nuclei.

\section{Conclusion}

We have presented an active surface model on an icosahedron subdivision grid structure, which is specially adapted to the segmentation of 2-sphere like objects. We have designed an external force field that is able to address the problems caused by wide-field microscopy imaging as well as the specific challenges of the segmentation of Drosophila Schneider cell nuclei. The segmentation results with our method were not only better and more reliable than the results found with standard methods, it was also possible to segment all correctly detected nuclei with the same parameter set, such that no further tedious manual adjustments were necessary. The model has shown its generalization ability in yielding very good results for the segmentation of the nucleoli inside these nuclei.

\section{Acknowledgments}

This study was supported by the Excellence Initiative of the German Federal and State Governments (EXC 294) 

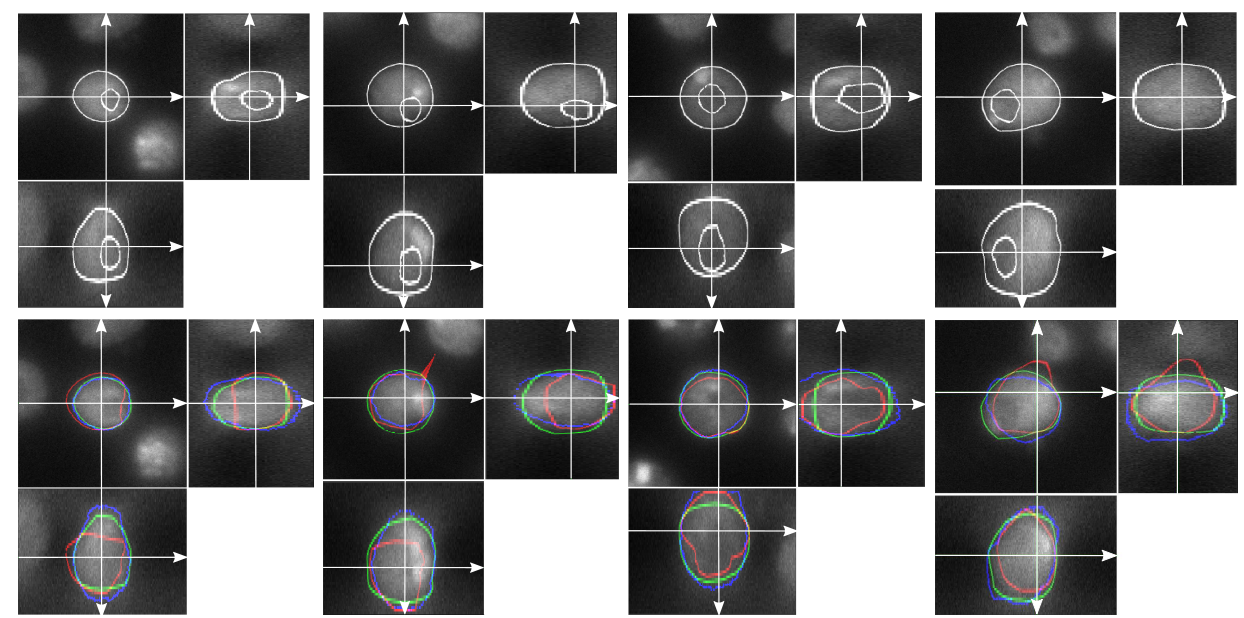

Fig. 7. (above) Orthogonal views of the segmentation results of four nuclei and nucleoli displayed in channel 1. The overall result is satisfying for nuclei as for nucleoli. (below) Segmentation results for the same nuclei segmented with our method (green), active surfaces with standard force field (red) and the region competition method from ITKSNAP (blue). The red contours are attracted by inner structures as well as neighboring cells and thus yield bad segmentation results, the blue contours are quite good, but elongated in z-direction, which is caused by the blurring in the dataset.

\section{References}

1. D.H. Ballard, Generalizing the hough transform to detect arbitrary shapes, Pattern Recognition,vol. 13, no 2, pp. 111-122, 1981.

2. O. Ronneberger, Q. Wang, H. Burkhardt, Fast and robust segmentation of spherical particles in volumetric data sets from brightfield microscopy, in Proc. of the ISBI (2008), 372-375.

3. J. Schulz, T. Schmidt, O. Ronneberger, H. Burkhardt, T. Pasternak, A. Dovzhenko, K. Palme, Fast scalar and vectorial grayscale based invariant features for $3 \mathrm{~d}$ cell nuclei localization and classification, in Proc. of the DAGM, Berlin, 2006.

4. J.-M. Geusebroek, B. Ter Haar Romeny, J. Koenderink, R. van den Boomgaard, P. van Osta, Color differential structure, Front-End Vision and Multi-Scale Image Analysis, Computational Imaging and Vision, vol. 27. Springer Netherlands, 2003.

5. C. Xu, J.L. Prince: Snakes, shapes, and gradient vector flow. IEEE Trans. Imag. Proc., vol 7, no. 3, 321-345, 1998.

6. J. Montagnat, H. Delingette, N. Ayache: A review of deformable surfaces: topology, geometry and deformation, Image and Vision Computing,19/14(2001) 1023-1040.

7. L. He, Z. Peng, B. Everding, X.Wang, C.Y. Han, K.L. Weiss, W.G. Wee: A comparative study of deformable contour methods on medical image segmentation. Image and Vision Computing,26/2 (2008), 141-163.

8. P.A. Yushkevich, J. Piven, C. Hazlett, H. Smith, G. Smith, R. Ho, S. Ho, J.C. Gee, G. Gerig: User-Guided 3D Active Contour Segmentation of Anatomical Structures: Significantly Improved Efficiency and Reliability, Neuroimage, 31/3(2006), 11161128. 